\title{
Immediate Recognition Memory for Wine
}

\author{
Andrew J. Johnson, ${ }^{1 *}$ Amy Volp ${ }^{2}$ and Christopher Miles ${ }^{3}$ \\ ${ }^{1}$ Psychology Research Centre, $\quad{ }^{2}$ Department of Psychology \\ ${ }^{3}$ School of Psychology, \\ Bournemouth University, and Behavioural Sciences, \\ Poole, \\ Coventry University, \\ Coventry, UK \\ Cardiff University, \\ Cardiff, \\ UK \\ UK
}

*Corresponding author

Address for Correspondence:

Psychology Research Centre

Bournemouth University

Poole House

Talbot Campus

Fern Barrow

Poole, UK

Tel: +0044 (0)1202965977

Email*: andjohnson@bournemouth.ac.uk

Word count: 3994 (excluding references but including the acknowledgement section)

\section{Acknowledgements}

The authors wish to thank Dr. John Williams for his advice regarding the selection of wines, and both Prof. Geoff Ward and an anonymous reviewer for their comments on the manuscript. 


\section{Abstract}

We describe a preliminary investigation concerning the short-term recognition memory function for gustatory stimuli (wines). In Experiment 1a, 24 non-expert wine drinkers completed a yes/no recognition task for 3-wine sequences. For the raw recognition scores, the serial position function comprised both primacy and recency. Recency did not, however, achieve significance for the $\mathrm{d}^{\prime}$ scores. In Experiment 1b, 24 participants completed the same yes/no recognition task for 3-visual matrix sequences. In contrast to Experiment 1a, the serial position function comprised recency and an absence of primacy. We argue that the presence of primacy for the wine sequences cannot be interpreted via a verbal labelling strategy, nor can it be interpreted via proactive interference from the first wine in the list on subsequent list items. The result suggests qualitative differences in the memory processing for gustatory and non-verbal visual stimuli.

Keywords: Working Memory; Gustatory; Serial Position 


\section{Introduction}

It is presently unclear how gustatory memory is represented within working memory. Whilst the traditional conceptualisation of working memory sub-division has focussed upon phonological and visual-spatial slave systems directed by an attention control system (e.g. Baddeley \& Hitch, 1974), this proposed structure neglects consideration of both the storage and manipulation of stimuli from other modalities (e.g. gustatory, olfactory, haptic, nonverbal auditory). It might be argued, in response, that memory for such stimuli are underpinned via verbal and/or visual recoding strategies. Indeed, there is evidence that verbal recoding strategies can facilitate, for example, gustatory memory (Hughson \& Boakes, 2009). However, other researchers have proposed auxiliary stores within the model that are stimulispecific (e.g. an olfactory-specific slave system, Andrade \& Donaldson, 2007) or multidimensional (Baddeley, 2000). The multi-dimensional episodic-buffer (Baddeley, 2000) has the potential to operate as a store for stimuli currently neglected by the modular slave systems

of the model. However, relative to the visual and verbal domains, there is a paucity of gustatory short-term memory studies and it remains unclear how gustatory memory might fit within a modular view of working memory. Specifically, it is ill-specified whether gustatory memory is reliant upon verbal recoding and, therefore, represented in the phonological loop, 
represented in multidimensional code within the episodic buffer, or held within a gustatory specific slave system.

A primary consideration for the examination of gustatory memory is the possibility of verbal re-coding. To the extent that gustatory stimuli are re-coded verbally, based upon either identification (e.g. "orange") or categorisation (e.g. "sour"), then patterns of memory performance might reflect verbal memory (for the gustatory labels), rather than gustatory memory, per se.

Research employing non-human animals (e.g. Reed, 2000) negates the concerns of verbal labelling, and has shown that water-deprived rats exhibit both primacy and recency effects for the recognition of 5-item sequences of novel-flavoured solutions. Reed (2000) speculates that primacy is underpinned via the positive hedonic association with the first list item (i.e. rats were water-deprived, with the first solution ascribed a more positive evaluation), whereas recency results from an accentuated positive evaluation due to its contiguity with satiation. An alternative approach to alleviate the possibility of verbal labelling in human participants is to employ stimuli that are hard to differentiate verbally (e.g. wine stimuli). Non-expert intermediate wine drinkers exhibit verbal overshadowing (Melcher \& Schooler, 1996), that is, 
verbal descriptions of wine stimuli disrupt recognition for the wine, arguably due to the perceptual, rather than verbal representation of wines; a finding consistent with the proposal that novice wine drinkers have an impoverished vocabulary for the description of wines (Hughson \& Boakes, 2002). The present study, therefore, presents participants with sequences of wine stimuli in order to examine serial position effects for gustatory stimuli, whilst minimising the possibilities for verbal recoding. Previous studies have examined serial position effects in taste preference. For instance, Mantonakis, Rodero, Lesschaeve \& Hastie (2009) reported a primacy advantage in the preference judgement of wines in sequences of $2,3,4$, and 5 items, despite the sequences comprising identical wines. It is conceivable that this primacy advantage for preference judgements might transfer to memory, since Reed (2000) speculates that the primacy and recency effects found with rat taste memory might be underpinned via hedonic factors (i.e. the first item is evaluated more positively). If a common mechanism determines preference and memory judgments, one might predict analogous serial position functions across the two paradigms.

For the current experiment participants were required to make single yes/no recognition judgements following the presentation of 3-wine sequences: a paradigm that typically 
produces a serial position function with single-item recency only (Hay, Smyth, Hitch \& Horton, 2007). For the current study the RI and ISI are equivalent (at 5s) and for single yes/no recognition, RIs of this duration are associated with a reduction in recency (e.g. Kerr, Avons \& Ward, 1999; Neath \& Knoedler, 1994). The effect of lengthened RIs on primacy is less clear; for instance, Neath and Knoedler (1994) reported an emergence of primacy, a finding not replicated by Kerr et al. (1999). In the present study we apply gustatory stimuli to the yes/no recognition task, and, specifically, test for the presence of primacy; a contentious finding for this task. Primacy is of particular interest since a first item benefit has already been reported for the preferential judgments of wines (Mantonakis et al., 2009), suggesting a potential first-item advantage for the memory of gustatory stimuli.

\section{Experiment 1a}

\section{Method}

Participants: Twenty-four (12 male, 12 female: mean age $=20$ years and 2 months) Psychology undergraduate volunteers from Coventry University participated in exchange for course credit. Participants were all occasional or regular consumers of alcohol and none self- 
reported expertise for wine recognition. Ethical approval was obtained from the Coventry University Ethics Committee.

Materials: A pilot study $(\mathrm{n}=8)$ revealed that for a yes/no recognition-probe paradigm comprising 4-item wine sequences, performance was at chance (i.e. 50\%). The present study, therefore, employed the same paradigm with 3-item wine sequences. A total of four wines were employed: two red (Australian Shiraz and Chilean Merlot) and two white (Australian Viogner and Chilean Sauvignon Blanc) presented at room temperature. In order to minimise visual cues, a number of precautions were employed: the wines were presented in opaque blue plastic cups with lids containing a single hole from which to drink, and participants were required to wear blue tinted goggles throughout the experiment.

Design: A within-participants design was employed in a single yes/no recognition-probe task. The independent variable of serial position comprised three levels (positions 1, 2, and 3). A testing protocol was produced for the experiment comprising 18 trials (9 test trials and 9 distractor trials). Recognition for items at each serial position was probed on three occasions. Within the testing protocol, each wine was assigned a number, i.e., 1, 2, 3 or 4 . These numbers were used in the protocol to define the composition of the sequence, the positioning of items within the sequence, and the item being tested (i.e. either a probe item from the list 
or a distracter at test). However, due to the number of trials (18) and the number of wines (4), it was not possible to fully counterbalance the serial position of each wine across the trials. Each wine was presented at the first, second, and third serial positions on 4, 5, or 6 occasions. In order to mitigate the effects of this variability, the assignment of each number (1-4) to each wine (Shiraz, Merlot, Viogner, and Sauvignon Blanc) was fully counterbalanced across the participants. For example, in the testing protocol for the first participant: 1 represented the Shiraz, 2 represented the Merlot, 3 represented the Viogner, and 4 represented the Sauvignon Blanc. In contrast, for the second participant: 1 represented the Shiraz, 2 represented the Merlot, 3 represented the Sauvignon Blanc, and 4 represented the Viogner etc. As a consequence, any effect of uneven presentation of wines for an individual participant is balanced across participants. This resulted in each participant receiving a unique presentation order, such that the effect of non-equivalent presentation of a particular wine is counterbalanced across participants.

Procedure: Participants were tested individually in a soundproofed laboratory and sat facing the experimenter across a table. A screen positioned between the participant and experimenter obscured the four wine samples from the participant. For each trial the experimenter presented a sequence of three wines to the participant. The participant briefly 
tasted the wine (approximately $5 \mathrm{~s}$ ) before discharging the wine into a spittoon. Participants were then given $5 \mathrm{~s}$ to clean their palate with a sip of water. This was followed by presentation of the next wine in the sequence. Following presentation of the third wine in the sequence (and successive water sip) participants were presented with the test wine which they both tasted and discharged. The test wine was either one of the three wines in the preceding sequence, or a fourth wine, not in the preceding sequence. Participants were required to judge whether the test wine was in the preceding list and respond verbally 'yes' or 'no', i.e. the task required a trial-specific familiarity judgment. Each trial was followed by an approximate 1 min. rest interval.

\section{Results and Discussion}

Because the single yes/no recognition paradigm is susceptible to a response-bias (i.e. inflation of correct recognition score via repetition of "yes"), both proportion correct and signal detection sensitivity $\left(d^{\prime}\right)$ scores are analysed $\left(d^{\prime}\right.$ is computed as described by Mondor \& Morin, 2004: z[hit]-z[false-alarm]). The current design (as in Mondor \& Morin, 2004) precludes the calculation of a false-alarm score for each serial position because participants were responding 'yes' to a distracter probe. Therefore, we cannot examine whether the erroneous 'yes' response was premised on a perception that the item was first, second, or 
third in the sequence. As a consequence, for the purposes of analysis the false-alarm value was equivalent across serial position calculations of $\mathrm{d}^{\prime}$.

The mean proportion correct recognition for the task was .70. Figure 1 shows the serial position functions for both the proportion correct (Figure 1a) and d' scores (Figure 1b). Both figures indicate a recognition advantage for the wine presented first and the wine presented last in the sequence.

Figure 1 about here please

Both the raw and $\mathrm{d}^{\prime}$ scores violated the assumption of normality (Shapiro-Wilk, $p<.05$ ), therefore, non-parametric tests were computed (Friedman's ANOVA: Bonferroni-corrected Wilcoxon Matched Pairs were used for post-hoc comparisons). A one-way repeated measures Friedman's ANOVA was computed on the proportion correct data and revealed a main effect of serial position, $\chi^{2}(2)=13.18, p=.001$. Consistent with the presence of both primacy and recency, post-hoc Bonferroni corrected comparisons $(\alpha=.017)$, revealed that recognition scores for both serial position $1(z=2.99, p=.003, r=.61)$ and serial position $3(z=2.61, p=$ 
$.009, r=.53$ ) exceeded that for serial position 2 . There was no difference in recognition scores between serial positions 1 and $3(z=1.03, p=.31, r=.21)$. The mean false alarm score in the yes/no recognition paradigm was .17 . To account for the possible effects of a response bias, the same analysis was conducted on the $\mathrm{d}^{\prime}$ scores and revealed a main effect of serial position, $\chi^{2}(2)=13.18, p=.001$. Following Bonferroni corrected comparisons, recognition score at serial position 1 was significantly greater than that at serial position $2(z=2.54, p=$ $.01, r=.52$ ). The recognition advantage for serial position 3 compared to serial position 2 approached (Bonferroni corrected) significance $(z=1.96, p=.05, r=.40)$. There was no significant difference between recognition scores at serial positions 1 and $3(z=1.19, p=.23$, $r=.24)$.

The experiment identified a primacy advantage in gustatory recognition that is consistent with the first item preference bias (Mantonakis et al., 2009). However, prior to any crossmodality comparisons with past studies, two important methodological differences are highlighted. First, for the present methodology, the stimulus set (4-wines) was presented repeatedly throughout the 18 trials. In contrast, both Kerr et al. (1999) and Neath and Knoedler (1999) employed trial unique stimuli. Repetition of stimuli therefore had the potential to encourage the build-up of proactive interference (PI) and/or increase verbal 
labelling opportunities not present in the Kerr et al. and Neath and Knoedler studies. Second, the requirement for participants to consume water during the ISI was idiosyncratic and it is possible that this requirement affected the serial position function (although it is unclear how sipping water during the ISI might underpin the development of primacy). Therefore, for Experiment $1 \mathrm{~b}$, the same recognition paradigm was applied to a 4-item set of hard-to-name visual stimuli. The comparison allows us to examine whether the serial position function obtained for Experiment 1a was a consequence of the increased familiarity with the stimulus set and/or the inter-stimulus interval activity of sipping water. This design thus enables a direct cross-modal comparison between gustatory and non-verbal visual recognition. Indeed, Ward, Avons, and Melling (2005) have proposed that the serial position function is determined by task constraints rather than the stimuli employed. Following this proposition, we predict primacy and recency for visual matrix sequences when subject to the same methodology as that employed in Experiment 1a. 


\section{Experiment 1b}

\section{Method}

Participants: Twenty-four (12 male, 12 female: mean age $=20$ years and 2 months)

undergraduate volunteers from Coventry University participated in exchange for course credit. None had participated in Experiment 1a and all participants reported normal or corrected to normal vision. Ethical approval was obtained from the Coventry University Ethics Committee.

Materials: A set of four $(4 \mathrm{x} 4)$ black and white matrices was selected from a corpus of 60 .

Each matrix comprised 8 white and 8 black squares (124mm x 124mm) configured randomly.

None represented an easy to name configuration (e.g. a letter name) and each matrix was visually distinguishable (see Figure 2).

Figure 2 about here please

Design: The same design as described for Experiment 1a was adopted. 
Procedure: The procedure followed closely that described for Experiment 1a. Participants sat facing a computer screen and were presented with 18 sequences each comprising 3 matrices. Each matrix was presented in the centre of the screen for $5 \mathrm{~s}$ using the presentation software Superlab 4.0. A $5 \mathrm{~s}$ ISI was employed during which participants were instructed to take a sip of water as described for Experiment 1a. Following the third matrix in the sequence, and successive water sip, participants were presented with a row of asterisks on the screen; this indicated the test phase. A single matrix was then presented and participants were instructed to press the letter 'o' if the matrix was judged to be in the preceding sequence and the letter ' $n$ ' if it was not. Participants commenced the next trial by pressing any key.

\section{Results and Discussion}

The mean proportion correct recognition score for the experiment was .66. Figure 3 demonstrates the serial position effects for the proportion correct and $\mathrm{d}^{\prime}$ scores across the three serial positions. Both indicate a final item recognition advantage, i.e. recency.

Figure 3 about here please 
Both the raw and $\mathrm{d}^{\prime}$ scores violated the assumption of normality; consequently, the same nonparametric tests as described for Experiment 1a were computed. A one-way repeated measures Friedman's ANOVA computed on the matrix proportion correct scores revealed a main effect of serial position, $\chi^{2}(2)=38.06, p<.001$. Consistent with the presence of recency, post-hoc Bonferroni corrected comparisons $(\alpha=.017)$, revealed that the recognition score for serial position 3 was significantly greater than that for both serial position $1(z=4.07, p<$ $.001, r=.83)$ and serial position $2(z=4.01, p<.001, r=.82)$. Recognition for serial position 2 was significantly greater than that at serial position $1(z=3.00, p=.003, r=.61)$, demonstrating an absence of primacy. The mean false-alarm rate in the yes/no recognition paradigm was .15 . To account for the effects of a response bias, the same analysis as described for Experiment 1a was computed on the $\mathrm{d}^{\prime}$ scores and revealed a main effect of serial position, $\chi^{2}(2)=38.06, p<.001$. Post-hoc tests revealed the same pattern of results as reported for proportion correct scores with recognition for serial position 3 significantly greater than that for both serial position $1(z=4.03, p<.001, r=.82)$ and serial position $2(z=$ 4.01, $p<.001, r=.80)$. Recognition for position 2 was significantly greater than that for serial position $1(z=2.70, p=.007, r=.55)$. 
The results of Experiment $1 \mathrm{~b}$ demonstrate recency with the absence of primacy. Notably, this function is qualitatively different to that reported for wines in Experiment 1a and, importantly, directly contradicts the Ward et al. (2005) proposition of cross-modal equivalence. Furthermore, the absence of primacy is consistent with the function found for abstract matrices following a $5 \mathrm{~s}$ RI (Kerr et al., 1999). This finding also militates against the possibility that the limited stimulus set and/or act of sipping water during the ISI was responsible for the development of primacy in Experiment 1a.

\section{General Discussion}

We report serial position effects for gustatory and visual recognition memory when applied to the yes/no recognition task. This task typically produces a function "not of a bow-shaped serial position curve but one with no primacy and only last-item recency" (Hay et al., 2007, p.176). The serial position function for abstract matrices supports this proposition; however, the serial position function for wine comprises both primacy and recency. This suggests differences in the working of gustatory and visual memory, particularly with respect to the memorial importance ascribed to the first item in the list and, additionally, contradicts the 
proposition by Ward et al. (2005) that the serial position function is determined by task type, rather than stimulus type. One speculative explanation for this observation is that gustatory memory operates qualitatively differently to that of other stimulus modalities. Indeed, previous work from our laboratory (Johnson \& Miles, 2009; Johnson, Cauchi \& Miles, 2013) has demonstrated that the serial position function for olfactory memory differs to that observed for the visual and auditory domains. These differences might suggest the existence of an olfactory-specific slave system within working memory (Andrade \& Donaldson, 2007), and the present data perhaps point towards similar dissociation for gustatory memory. One might speculate that the different requirements for the processing of the chemical senses have necessitated a functionally different memory system to that of 'far senses' such as vision.

We now consider, and dismiss, a number of alternative explanations for the observation of primacy for wines. First, a set of four wines might have facilitated verbal labelling of the wines, with verbal rehearsal of early list items promoting superior recognition (although verbal rehearsal is not the only explanation for primacy, Ward, Tan \& Grenfall-Essam, 2010). Against this account, we argue that our selection of non-expert wine drinkers as participants, who are known to have an impoverished descriptive vocabulary for the stimuli (Hughson \& Boakes, 2002), minimised verbal labelling possibilities. Second, despite the restriction of 
visual cues, participants may have been able to verbally categorise the wines by colour (i.e. "red" or "white"). However, there was no evidence of recognition improvement across trials (first half and second half performance $=71.30 \%$ and $69.44 \%$, respectively, $t<1$ ) as one might expect with the development of a colour labelling strategy. More importantly, even if participants utilised a colour based verbal labelling strategy, it is not clear how such a strategy might systematically benefit a particular position. That is, if participants were able to utilise the red/white verbal categorisation strategy, this would be most beneficial in situations where the test wine was the same colour as two wines in the preceding list (e.g. there were two red wines in the to-be-remembered list and the test wine was one of those two red wines). Assuming participants have deduced the existence of two red and two white wines in the study, participants would respond "yes" to the test wine since they know that the test wine is red and both were in the preceding sequence. However, due to balancing of sequence compositions across participants, the positioning of the two same-colour wines in the above example is equally distributed across the three serial positions. Consequently, the advantage of this strategy would act to inflate overall recognition scores but would not impact a particular serial position and thus, cannot explain the qualitative difference with abstract matrices (i.e. the development of primacy). 
Third, the perceptual properties of the chemical stimulus may persist and thereby contaminate subsequent list items (termed 'generalization decrement' by Reed, 2000) provoking withintrial PI. Since only the first list item is free of such perceptual contamination, this might explain the memory advantage for that item. We suggest that this account is unlikely for three reasons. First, as argued by Reed (2000), such a contamination account does not predict a recency benefit because of (proactive) perceptual interference from the preceding list items. However, there was evidence of a recency advantage in both the present study and in Reed (2000). Second, the consumption of water during the ISI was employed to 'cleanse the palette' and hence minimise the possibility of perceptual contamination. Indeed, Reed (2000) reported accentuated recency following the addition of water consumption during the ISI, indicating that this intervention is effective in mitigating perceptual contamination. Third, we find no evidence of a contamination/interference increase across the experimental trials; as reported earlier, there was no significant difference between first and second half recognition performance.

Fourth, Reed (2000) suggests that for gustatory memory, both primacy and recency might be explained via hedonic factors. Specifically, since in his studies the rats were water-deprived, the first solution in the sequence will be associated with accentuated pleasantness, and, 
therefore, enhanced memory. The final solution in the sequence will be associated with satiation, and, therefore, be ascribed greater salience in memory. We argue that this hedonic account cannot explain the serial position function observed in Experiment 1a. Our participants were not water-deprived and, therefore, the wines should not be associated with satiation. Moreover, unlike the single-trial methodology employed by Reed (2000), our participants completed 18 trials. Therefore, the hedonic account can accommodate trial 1 performance only, with such hedonic effects diluted across the subsequent 17 trials.

Fifth, it could be argued that our results simply reflect differences in stimulus discriminability (although, importantly, recognition levels did not differ significantly: mean proportion correct for wines and matrices $=.70$ and .66 , respectively, $p=.26$ ). Hay et al. (2007) used yes/no recognition to test the predictions of the Scale-Invariant, Memory, Perception, and Learning (SIMPLE) model (Brown, Neath \& Chater, 2007). One component of this model focusses upon the 'psychological distinctiveness' of the stimuli within a sequence. Hay et al. (2007) employed high (faces) and low (inverted faces and matrices) psychologically distinctive stimuli and showed that, although distinctiveness increased the number of positions for which recency was extended, it did not promote the development of primacy. By extrapolation, we argue that the cross-modal difference in primacy reported here cannot be explained by 
differences in psychological distinctiveness for wines and matrices. Nevertheless, Hay et al.'s speculation on the absence of primacy might explain the cross-stimuli differences in primacy reported in the present study. They suggest that primacy is absent in the yes/no recognition task because the close temporal proximity of trials produces between-trial PI: interference from earlier trials is proposed to affect memory for the first item of successive trials. Only under conditions of longer inter-trial intervals is the first item released from PI and, as a consequence, primacy is observed. In Experiment 1a, there was an approximate 1 minute inter-trial interval for the wines. In contrast, Experiment $1 \mathrm{~b}$ was self-paced between trials and some may have proceeded to the next trial with a limited rest interval. As a consequence, the absence of primacy for matrices might be explained by PI. It must be stressed, however, that this between-trial PI account fails to explain how primacy is observed in serial reconstruction tasks for which similarly short inter-trial intervals are employed (e.g. Ward et al., 2005).

In summary, the current preliminary data set indicates that gustatory memory operates differently to that for other stimuli. However, further empirical work is required in order to establish whether differences in between-trial PI can account for this disparity. 


\section{References}

Andrade, J., \& Donaldson, L. (2007). Evidence for an olfactory store in working memory. Psychologia, 50, 76-89. doi.org/10.2117/psysoc.2007.76

Baddeley, A. (2000). The episodic buffer: a new component of working memory? Trends in Cognitive Sciences, 4(11), 417-423. doi.org/10.1016/S1364-6613(00)01538-2

Baddeley, A.D., \& Hitch, G.J. (1974). Working memory. In G.A. Bower (Eds). The Psychology of Learning and Motivation (pp. 47-89). London: Academic Press

Brown, G.D.A., Neath, I., \& Chater, N. (2007). A temporal ratio model of memory. Psychological Review, 114, 539-576. doi:10.1037/0033-295X.114.3.539

Hay, D.C., Smyth, M.M., Hitch, G.J., \& Horton, N.J. (2007). Serial position effects in shortterm visual memory: A SIMPLE explanation? Memory and Cognition, 35, 176-190. doi:10.3758/BF03195953

Hughson, A.L., \& Boakes, R.A. (2002). Perceptual and cognitive aspects of wine expertise. Australian Journal of Psychology, 53(2), 103-108. doi:10.1080/00049530108255130 
Hughson, A.L., \& Boakes, R.A. (2009). Passive perceptual learning in relation to wine: Short-term recognition and verbal description. Quarterly Journal of Experimental Psychology, 62(1), 1-8. doi:10.1080/1747021802214890

Johnson, A.J., \& Miles, C. (2009). Single-probe serial position recall: evidence of modularity for olfactory, visual and auditory short-term memory. Quarterly Journal of Experimental Psychology, 62(2), 267-275. doi:10.1080/17470210802303750

Johnson, A.J., Cauchi, L., \& Miles, C. (2013). Hebbian learning for olfactory sequences. Quarterly Journal of Experimental Psychology, 66(6), 1082-1089. doi:10.1080/17470218.2012.729068

Kerr, J.R., Avons, S.E., \& Ward, G. (1999). The effect of retention interval on serial position curves for item recognition of visual patterns and faces. Journal of Experimental Psychology: Learning Memory and Cognition, 25(6), 1475-1494. doi:10.1037/02787393.25.6.1475

Mantonakis, A., Rodero, P., Lesschaeve, I., \& Hastie, R. (2009). Order in choice: Effects of serial position on preferences. Psychological Science, 20(11), 1309-1312. 
Melcher, J.M., \& Schooler, J.W. (1996). The misremembrance of wines past: verbal and perceptual expertise differentially mediate verbal overshadowing of taste memory. Journal of Memory and Language, 35, 231-245. doi:10.1006/jmla.1996.0013

Mondor, T.A., \& Morin, S.R. (2004). Primacy, recency and suffix effects in auditory shortterm memory for pure tones: Evidence from a probe recognition paradigm. Canadian Journal of Experimental Psychology, 58(3), 206-219. doi:10.1037/h0087445

Neath, I. (1993). Distinctiveness and serial position effects in recognition. Memory and Cognition, 21(5), 689-698. doi:103758/BF03197199

Neath, I., \& Knoedler, A.J (1994). Distinctiveness and Serial Position Effects in recognition and sentence processing. Journal of Memory and Language, 33, 776-795. doi:10.1006/jmla.1994.1037

Reed. P. (2000). Rats' memory for serially presented flavors: Effects of interstimulus interval and generalization decrement. Animal Learning \& Behavior, 28(2), 136-146.

Ward, G., Avons, S.E., \& Melling, L. (2005). Serial position in curves in short-term memory: Functional equivalence across modalities. Memory, 13(3/4), 308-317. doi:10.1080/09658210344000279 
Ward, G., Tan, L., \& Grenfall-Essam, R. (2010). Examining the relationship between free recall and immediate serial recall: the effects of list length and output order. Journal of Experimental Psychology: Learning, Memory, and Cognition, 36(5), 1207-1241. doi:10.1037/a0020122

Wright, A.A., Santiago, H.C., Sands, S.F., Kendrick, D.F., \& Cook, R.G. (1985). Memory processing of serial lists by pigeons, monkeys and people. Science, 229, 287-289. 
Figure 1

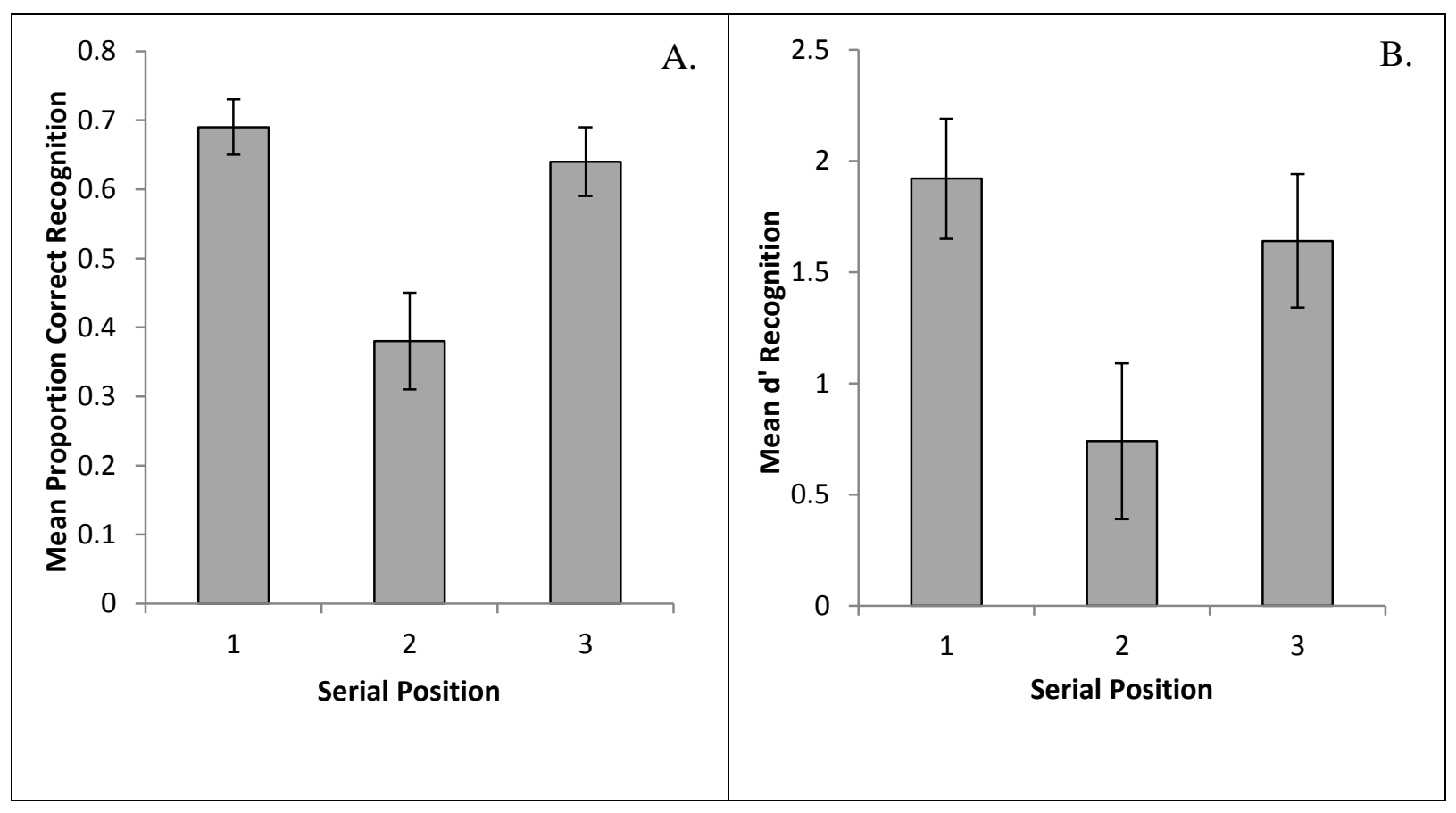


Figure 2
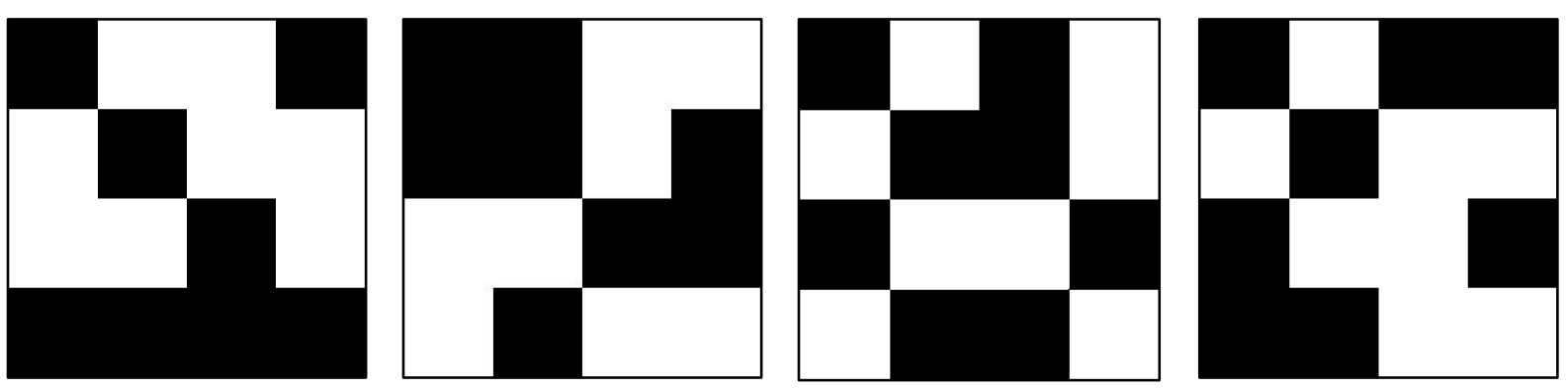
Figure 3

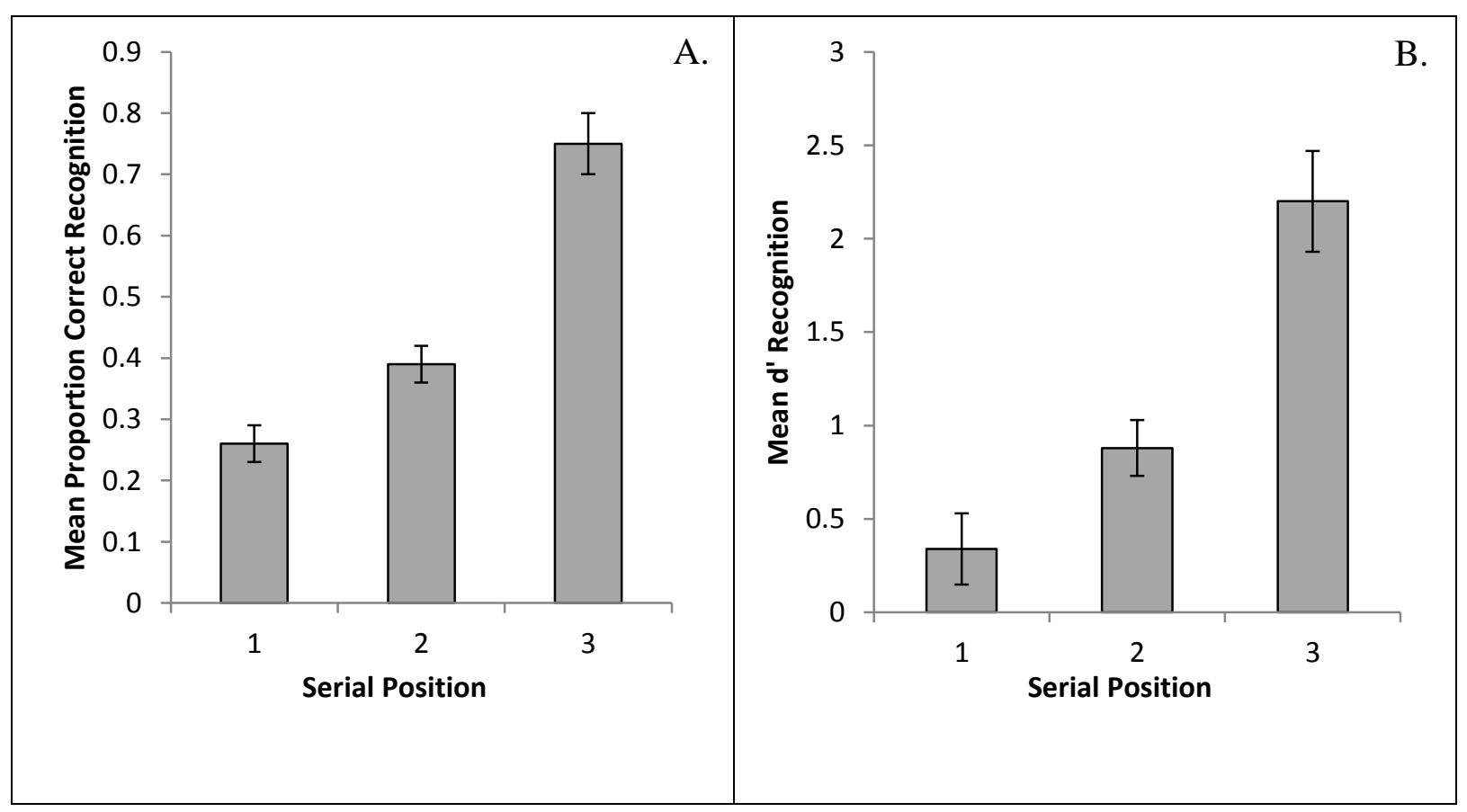




\section{Figure Legends}

Figure 1. Mean correct recognition (a) and d' (b) scores for the wines as a function of serial position (1-3). Error bars denote the mean standard error.

Figure 2. Matrix (4x4) stimuli employed in Experiment $1 \mathrm{~b}$.

Figure 3. Mean correct recognition (a) and d' (b) scores for the visual matrices as a function of serial position (1-3). Error bars denote the mean standard error. 